\title{
Sepsis in internal wards: results of an Italian multicenter prospective study
}

\author{
Matteo Giorgi-Pierfranceschi, ${ }^{1}$ Francesco Dentali ${ }^{2}$ \\ ${ }^{1}$ Emergency Department, Hospital of Val d'Arda, AUSL Piacenza; ${ }^{2}$ Department of Medicine, University of Insubria, Varese, \\ Italy
}

\begin{abstract}
Sepsis is a frequent inflammatory disease with a high mortality and morbidity rate. Most of the data about epidemiology, management and prognosis of patients with sepsis came mainly from studies conducted within Intensive Care Units (ICUs). A consistent number of studies suggest that a proportion of patients with sepsis and severe sepsis are admitted to internal medicine units, and not transferred to an ICU. In this article, we presented the data of an Italian study, a multicenter study, evaluating consecutive patients, with an objective diagnosis of sepsis treated in internal medicine units.
\end{abstract}

\section{Introduction}

Sepsis is a frequent inflammatory disease with a high mortality and morbidity rate. ${ }^{1}$ Over the past few years, a number of different studies have reported an increasing incidence of this disease ${ }^{2-4}$ that overcomes the possibility to treat these patients exclusively in Intensive Care Units (ICU).

Nevertheless, most of the data about epidemiology, management and prognosis of patients with sepsis came mainly from studies conducted within ICUs. ${ }^{25,6}$ A consistent number of studies suggest that a proportion of patients with sepsis and severe sepsis are admitted to internal medicine units, and not transferred to an ICU.7,8

We had only scarce data on the epidemiology and management of sepsis in the internal medicine setting. ${ }^{8}$

Studies on the clinical history of patients with sepsis treated outside an ICU are extremely limited, and most of them have been conducted before the implementa-

Correspondence: Matteo Giorgi-Pierfranceschi, Emergency Department, Hospital of Val d'Arda, AUSL Piacenza, via Roma 35, 29017 Fiorenzuola d'Arda (PC), Italy.

Tel.: +39.0523.989605.

E-mail: m.giorgi@ausl.pc.it

Key words: Sepsis; multicenter study; internal wards.

Received for publication: 13 October 2016.

Accepted for publication: 20 October 2016.

This work is licensed under a Creative Commons Attribution NonCommercial 4.0 License (CC BY-NC 4.0).

(C) Copyright M. Giorgi-Pierfranceschi and F. Dentali, 2016 Licensee PAGEPress, Italy

Italian Journal of Medicine 2016; 10:272-274

doi:10.4081/itjm.2016.791 tion of recent international guidelines on the management of severe sepsis and septic shock. They reported a higher mortality rate in patients with sepsis not admitted or with a delayed admission to an ICU. $., 9,10$

Recently, to address this knowledge gap, we conducted a prospective multicenter study, evaluating consecutive patients, with an objective diagnosis of sepsis treated in internal medicine units. ${ }^{11}$

This study was conducted in thirty-one internal medicine units from 14 different Italian regions.

Consecutive patients with an objective diagnosis of sepsis, admitted to these units from March 1, 2012, to December 31, 2012, were included. According to the definitions of the American College of Chest Physicians/Society of Critical Care Medicine Consensus Conference, sepsis was defined as infection, plus 2 systemic inflammatory response syndrome criteria. ${ }^{12,13}$ Severe sepsis was defined as sepsis plus at least 1 organ dysfunction, except when that organ dysfunction was already present $48 \mathrm{~h}$ before the onset of sepsis. ${ }^{14}$ Septic shock was defined as sepsis plus either hypotension refractory to intravenous fluids (defined as persistent hypotension or a requirement for vasopressors after the administration of an intravenous fluid bolus) or hyperlactatemia. ${ }^{14}$ Organ dysfunction was defined in accordance with commonly established criteria. ${ }^{1}$ Five hundred and thirty-three septic patients (mean age 73.3 years, $50.8 \%$ men) were included representing the $1.78 \%$ [ $95 \%$ confidence interval $(\mathrm{CI})$ $1.63,1.95]$ of the admissions to the internal medicine units in the same period; 316 patients $(59.3 \%)$ had a severe sepsis, and 17 (3.2.\%) septic shock upon presentation. Sepsis most frequently came from genitourinary $(30.8 \%)$, respiratory tract $(26.5 \%)$, and gastrointestinal tract (18.9\%), and 62 patients $(11.6 \%)$ had a primary bacteremia. Almost all patients $(94.2 \%)$ had at least 1 comorbidity: cardio- or cerebrovascular 
disease $(63.4 \%)$ and diabetes $(30.8 \%)$ were the most frequent comorbidities. Antibiotic therapy was started within $1 \mathrm{~h}$ after clinical suspicion of sepsis in $337 \mathrm{pa}-$ tients $(75.9 \%)$. There were 626 blood cultures positive: $313(50.0 \%)$ for Gram-positive bacteria, 293 $(46.8 \%)$ for Gram-negative bacteria, and only 20 (3.2\%) for fungi. Escherichia coli $(29.4 \%)$ was the most frequent isolated followed by Staphylococcus aureus (12.0\%). Using a multivariate model, clinical presentation as severe sepsis or septic shock [odds ratio (OR) 4.41 95\% CI 1.93, 10.05], immune system weakening (OR 2.10, 95\% CI 1.12, 3.94), presence of active solid cancer (OR 2.14, 95\% CI 1.16, 3.94), and age (OR 1.03 per year, 95\% CI 1.01, 1.06) were significantly associated with an increased mortality risk during hospitalization, whereas blood culture positive for $E$. coli was significantly associated with a reduced mortality risk (OR $0.46,95 \%$ CI $0.24,0.88)$.

\section{Discussion}

In our study, we collected information on a consistent number of patients with sepsis and with positive blood culture who were consecutively admitted to an internal medicine unit. The in-hospital mortality of this population appeared to be in line with the results of recent studies that have evaluated the prognosis of patients with sepsis ${ }^{15,16}$ and that show a declining trend in the hospital mortality rate of these patients over the past few years. ${ }^{17}$

Our results may be of clinical relevance for clinicians since, to date, only a few studies have provided information on the epidemiology and clinical history of patients with sepsis diagnosed and treated outside an ICU..$^{8}$ In fact, a significant proportion of patients with sepsis and even with severe sepsis are admitted to an internal medicine unit and not transferred to an ICU. ${ }^{7}$ Results of recent studies clearly show that the use of central venous catheterization to monitor central venous pressure and central venous oxygen saturation to guide the administration of intravenous fluids, vasopressors, packed red-cell transfusions, and dobutamine, did not modify the mortality and morbidity rate of patients with severe sepsis or septic shock. ${ }^{18,19}$

Thus, these results seem to suggest that some patients may be treated safely, without the need of a continuous invasive monitoring, in a less intensive unit provided that an adequate therapy was rapidly administered. ${ }^{20}$ Interestingly, other recent studies conducted on patients with sepsis admitted to ICU showed similar results, confirming the validity of our findings. ${ }^{21}$ However, information on clinical and easily assessable factors, potentially associated with clinical deterioration and short-term mortality, remains critical as a delayed transfer to an ICU of these patients seems to be associated with a worse short-term prognosis. ${ }^{21}$
Besides the clinical presentation as severe sepsis or septic shock other factors such as the presence of active solid cancer or of immune system weakening and age appeared associated with an increased mortality risk during hospitalization. As no previous study has specifically assessed the role of potential risk factors for short-term mortality in patients with sepsis admitted to an internal medicine unit, it was not possible to compare our results with preceding literature in this field.

Information on the most frequent infection in different settings is crucial in order to establish the most appropriate treatment of patients with sepsis. Grampositive bacteria were slightly more prevalent than Gram-negative bacteria (50.0\% vs $46.8 \%)$ and only $3.2 \%$ of blood cultures were positive for fungi. Escherichia coli was the most frequent bacterium isolated followed by $S$. aureus, S. epidermidis and E. faecalis (7.4\%). In previous studies, Gram-positive infections appeared to be more frequent than Gram-negative. ${ }^{4}$ However, more recently, in a study involving 14,000 ICU patients in 75 countries, Gram negative bacteria were isolated in $62 \%$ of patients with severe sepsis with positive cultures, Gram-positive bacteria in $47 \%$, and fungi in $19 \% .{ }^{5}$ At least 1 antibiotic resistance was present in almost $80 \%$ of the isolated at the blood culture and antibiotic resistance affected almost half of the empiric treatments. Interesting, E. coli infection was significantly associated with a reduced mortality risk in comparison to other bacteria at univariate analysis. Escherichia coli is very common in urinary tract infection sepsis that has a better prognosis in comparison to pneumonia-mediated sepsis. ${ }^{5}$ However, this association remained significant also at multivariate analysis that took in consideration among the other variables also the origin of infection. Finally, different from the results of a recent study on 327 adult septic patients admitted to ICU, primary bacteremia was not associated with an increased mortality risk in our population. ${ }^{22}$ Differences in the selection of the population may explain these different results. Alternatively, as only a small number of patients had a primary bacteremia in the 2 populations, these different results may be due to chance. Our study has strengths and limitations. First, only patients with positive blood cultures were included in our study. Although this may be considered to be a selection and thus the patients may not be representative of general patients with sepsis admitted to an internal medicine unit, with this approach we were able to exclude all those patients without a clear diagnosis of sepsis, increasing the validity of our results. Second, information on recent hospitalization was not available in almost $9 \%$ of included patients. Although the lack of this information in a non-negligible proportion of patients may affect the validity of our results, however the repetition of the multivariate analysis after the exclusion of this param- 
eter gave similar results minimizing this possibility. In addition, $<1 \%$ of data of all the others were missing. Third, results on the mortality rate of the subgroup of patients with septic shock should be interpreted with extreme caution as an extremely low number of patients with this condition $(n=17)$ have been included in our study. Finally, there may be important differences in the epidemiology, management and clinical history of sepsis among the involved centers. However, due to the relative low number of patients included in each center, this could not be accurately explored.

\section{Conclusions}

In conclusion, the in-hospital mortality rate of septic patients admitted to internal medicine units appeared in line with recent reports of the literature in ICU setting. Besides the clinical presentation as severe sepsis or septic shock, other factors appeared to be helpful in defining the short-term prognosis of septic patients and may be used to define the adequate management of these patients. However, other larger prospective studies are warranted to confirm our preliminary findings.

\section{References}

1. Angus DC, van der Poll T. Severe sepsis and septic shock. N Engl J Med. 2013;369:840-51.

2. Angus DC, Linde-Zwirble WT, Lidicker J, et al. Epidemiology of severe sepsis in the United States: analysis of incidence, outcome, and associated costs of care. Crit Care Med 2001;29:1303-10.

3. Sundararajan V, Macisaac CM, Presneill JJ, et al. Epidemiology of sepsis in Victoria, Australia. Crit Care Med 2005;33:71-80.

4. Martin GS, Mannino DM, Eaton S, et al. The epidemiology of sepsis in the United States from 1979 through 2000. N Engl J Med. 2003;348:1546-54.

5. Vincent JL, Rello J, Marshall J, et al. EPIC II Group of Investigators. International study of the prevalence and outcomes of infection in intensive care units. JAMA 2009;302:2323-9.

6. Dellinger RP, Levy MM, Rhodes A, et al. Surviving Sepsis Campaign Guidelines Committee including The Pediatric Subgroup. Surviving Sepsis Campaign: international guidelines for management of severe sepsis and septic shock, 2012. Intensive Care Med 2013;39:165-228.

7. Esteban A, Frutos-Vivar F, Ferguson ND, et al. Sepsis incidence and outcome: contrasting the intensive care unit with the hospital ward. Crit Care Med 2007;35: 1284-9.
8. Vardi M, Ghanem-Zoubi NO, Bitterman H, et al. Sepsis in nonagenarians admitted to internal medicine departments: a comparative study of outcomes. QJM. 2013; 106:261-6.

9. Sprung CL, Geber D, Eidelman LA, et al. Evaluation of triage decisions for intensive care admission. Crit Care Med 1999;27:1073-9.

10. Young MP, Gooder VJ, McBride K, et al. Inpatient transfers to the intensive care unit: delays are associated with increased mortality and morbidity. J Gen Intern Med. 2003;18:77-83.

11. Mazzone A, Dentali F, La Regina M, et al. Clinical features, short-term mortality, and prognostic risk factors of septic patients admitted to internal medicine units: results of an Italian Multicenter Prospective Study. Medicine (Baltimore) 2016;95:e2124.

12. Dellinger RP, Levy MM, Carlet JM, et al. Surviving Sepsis Campaign: international guidelines for management of severe sepsis and septic shock: 2008. Intensive Care Med 2008;34:17-60.

13. Bone RC, Sibbald WJ, Sprung CL. The ACCP-SCCM consensus conference on sepsis and organ failure. Chest 1992;101:1481-3.

14. Levy MM, Fink MP, Marshall JC, et al. International Sepsis Definitions Conference. $2001 \mathrm{SCCM} / \mathrm{ES} / \mathrm{CM} / \mathrm{ACCP} /$ ATS/SIS International Sepsis Definitions Conference. Intensive Care Med 2003;29:530-8.

15. Lagu T, Rothberg MB, Shieh MS, et al. Hospitalizations, costs, and outcomes of severe sepsis in the United States 2003 to 2007. Crit Care Med 2012;40:754-61.

16. Kumar G, Kumar N, Taneja A, et al. Milwaukee Initiative in Critical Care Outcomes Research Group of Investigators. Nationwide trends of severe sepsis in the 21st century (2000-2007). Chest 2011;140:1223-31.

17. Stevenson EK, Rubenstein AR, Radin GT, et al. Two decades of mortality trends among patients with severe sepsis: a comparative meta-analysis. Crit Care Med 2014:42:625-31.

18. Jones AE, Shapiro NI, Trzeciak S, et al. Lactate clearance vs central venous oxygen saturation as goals of early sepsis therapy: a randomized clinical trial. JAMA 2010;303:739-46.

19. Yealy DM, Kellum JA, Huang DT, et al. for the ProCESS Investigators. A randomized trial of protocolbased care for early septic shock. N Engl J Med 2014; 370:1683-93.

20. Lilly CM. Protocol-based care for early septic shock. N Engl J Med. 2014;371:386-7.

21. Keegan MT, Gajic O, Afessa B. Severity of illness scoring systems in the intensive care unit. Crit Care Med 2011;39:163-9.

22. Mansur A, Klee Y, Popov AF, et al. Primary bacteraemia is associated with a higher mortality risk compared with pulmonary and intra-abdominal infections in patients with sepsis: a prospective observational cohort study. BMJ Open 2015;5:e006616. 